\title{
CODIMENSION ONE MINIMAL FOLIATIONS WHOSE LEAVES HAVE FUNDAMENTAL GROUPS WITH THE SAME POLYNOMIAL GROWTH
}

\author{
TOMOO YOKOYAMA
}

\begin{abstract}
Let $\mathcal{F}$ be a transversely orientable codimension one minimal foliation without vanishing cycles of a manifold $M$ and $k \in \mathbb{Z}_{>0}$. We show that if the fundamental group of each leaf of $\mathcal{F}$ has polynomial growth of degree $k$, then the foliation $\mathcal{F}$ is without holonomy.
\end{abstract}

\section{INTRODUCTION}

In YT08, we have considered the following question: how is the minimal foliation without null homotopic closed transversals if the fundamental group of each leaf is isomorphic to an elementary group?

We have showed the following result in the paper: If the fundamental groups of all leaves are isomorphic to $\mathbb{Z}$, then the foliation is without holonomy. But if the fundamental groups of the leaves are "complicated", then the foliation can have nontrivial holonomy. In particular, there is such a foliation without vanishing cycles (e.g. Example 3.2. [YT08). In this paper, we consider the above question for the minimal foliations without vanishing cycles and obtain the following result.

Theorem 1.1. Let $\mathcal{F}$ a codimension one transversely orientable $C^{0}$ minimal foliation without vanishing cycles of a manifold $M$ and $k$ a nonnegative integer. If all fundamental groups of every leaves of $\mathcal{F}$ have the same polynomial growth of degree $k$, then the foliation $\mathcal{F}$ is without holonomy.

Note that a finitely generated group has polynomial growth if and only if it is virtually nilpotent G81. Notice that we do not require that the manifolds are compact.

\section{Preliminaries}

First, we recall a vanishing cycle in the sense of Novikov [N65.

Definition 2.1. Let $\mathcal{F}$ be a foliation of a manifold $M$. A loop $\gamma$ on the leaf $L \in F$ is called a vanishing cycle if there is a mapping $F: S^{1} \times[0,1] \rightarrow M$ such that arcs $F(x,[0,1])$ for every $x \in S^{1}$ are transverse to $\mathcal{F}$, each loop $F\left(S^{1}, t\right)$ for any $t \in[0,1]$ is contained in some leaf $L_{t},\left[F\left(S^{1}, 0\right)\right]=[\gamma] \neq 1 \in \pi_{1}(L)$, and $\left[F\left(S^{1}, s\right)\right]=1 \in \pi_{1}\left(L_{s}\right)$ for all $\left.\left.s \in\right] 0,1\right]$.

We define a trivial fence as follows [Y09].

The author is partially supported by the JST CREST Program at Creative Research Institution, Hokkaido University. 
Definition 2.2 (Trivial fences). Let $\mathcal{F}$ be a transversely oriented foliation of a manifold $M$. For a compact subset $K$ of a leaf $L_{0}$ of $\mathcal{F}$, an embedding $F: K \times$ $[0,1] \longrightarrow M$ is called a positive trivial fence if $F(K \times\{t\})$ is contained in a leaf $L_{t}$ of $\mathcal{F}, F \mid K \times\{0\}$ is the inclusion $K \subset L_{0}$ and $F(\{x\} \times[0,1])$ is transverse to $\mathcal{F}$.

Remark 2.3. For an arcwise connected compact subset $K$ of a leaf $L$, a positive trivial fence on $K$ exists if and only if the holonomy on the positive side along any loop in $K$ is trivial.

We define polynomial growth for finitely generated groups.

Definition 2.4. Let $G$ a finitely generated group and $\mathcal{G}$ be a generating set of $G$. The word length function $f_{G}: G \rightarrow \mathbb{Z}_{\geq 0}$ on $G$ with respect to $\mathcal{G}$ is given by $f_{G}(e):=0$ and $f_{G}(g):=\min \left\{n \in \mathbb{Z}_{>0} \mid g=g_{1} \cdots g_{n}\right.$ for some $\left.g_{i} \in \mathcal{G} \cup \mathcal{G}^{-1}\right\}$ for any nontrivial element $g \in G$. Let $G_{m}$ be the set of the elements of $G$ whose word lengths are at most $m$. We say that $G$ has polynomial growth of degree at least some nonnegative integer $k$ if there is a positive constant $\alpha \in \mathbb{R}_{>0}$ such that $\# G_{m} \geq \alpha m^{k}$ for all $m \in \mathbb{Z}_{\geq 0}$. If the group $G$ does not have polynomial growth of degree at least $k+1$, then $G$ is said to have polynomial growth of degree $k$.

Note that the definition of polynomial growth is independent of the choice of generating sets. To prove the main theorem, the following statement will be helpful.

Lemma 2.5. Let $G$ be a group with polynomial growth of degree $k \in \mathbb{Z}_{\geq 0}$ and $H \leq G$ subgroup of $G$ with the same growth. Then, for any $g \in G$, there is a positive integer $n \in \mathbb{Z}_{>0}$ such that $g^{n} \in H$.

Proof. Let $\mathcal{G}$ be a generating set of $G$ and $\mathcal{H}$ a generating set of $H$ with $\mathcal{H} \subseteq \mathcal{G}$. We define the word lengths on $G$ and $H$ by these generating sets. Let $G_{m}$ be the set of the elements of $G$ whose word lengths are at most $m$ and $H_{m}$ the set of the elements of $H$ whose word lengths are at most $m$. By contradiction, suppose that there exists $g_{0} \in G$ such that $g_{0}^{n} \notin H$ for any $n \in \mathbb{Z}_{>0}$. With out loss of generality, we may assume that $g_{0} \in \mathcal{G}$.

Claim 1. For $n, m \in \mathbb{Z}$ and $h, h^{\prime} \in H, g_{0}^{n} h^{\prime}=g_{0}^{m} h$ if and only if $n=m$ and $h^{\prime}=h$. In particular, $g_{0}^{j} H_{k} \cap g_{0}^{j^{\prime}} H_{k^{\prime}}=\emptyset$ for any $j \neq j^{\prime}$.

Indeed, if $n=m$ and $h^{\prime}=h$, then obviously $g_{0}^{n} h^{\prime}=g_{0}^{m} h$. Conversely, suppose that $g_{0}^{n} h^{\prime}=g_{0}^{m} h$. If $n \neq m$, then $H \ni h^{\prime} h^{-1}=g_{0}^{m-n} \notin H$ which is a contradiction. Thus $n=m$ and so $h^{\prime}=h$.

Since $G_{m} \supseteq \bigsqcup_{j=0}^{m} g_{0}^{j} H_{m-j}$, we have

$$
\# G_{m} \geq \sum_{j=0}^{m} \# g_{0}^{j} H_{m-j} .
$$

By the definition of polynomial growth, there exists $\alpha \in \mathbb{R}_{>0}$ such that $\# H_{m} \geq \alpha m^{k}$ for all $m \in \mathbb{Z}_{\geq 0}$. Thus

$$
\sum_{j=0}^{m} \# g_{0}^{j} H_{m-j}=\sum_{j=0}^{m} \# H_{j} \geq \alpha \cdot \sum_{j=0}^{m} j^{k} .
$$

Therefore $\# G_{m} \geq \alpha \cdot \sum_{j=0}^{m} j^{k}$. The well known fact that $\sum_{j=1}^{M} j^{k} \approx \frac{1}{k+1} M^{k+1}$ for sufficiently large $M \gg k$, implies that there is a positive constant $c \in \mathbb{R}_{>0}$ such that $\sum_{j=0}^{m} j^{k} \geq c \cdot m^{k+1}$ for all $m \in \mathbb{Z}_{\geq 0}$. Then we have $\# G_{m} \geq \alpha c \cdot m^{k+1}$ for all 
$m \in \mathbb{Z}_{\geq 0}$. Therefore the growth of $G$ is more than $k$. This contradicts with the hypothesis.

\section{A Key LEMma AND THE PROOF OF THE MAIN THEOREM}

The following key lemma will complete the proof of main theorem. Denote by $\operatorname{gr}(G)$ the degree of the polynomial growth of a group $G$.

Lemma 3.1. Let $\mathcal{F}$ a codimension one transversely orientable minimal $C^{0}$ foliation on a manifold $M$. If there is a leaf $L_{0} \in \mathcal{F}$ without holonomy such that the induced homomorphism $i_{*}: \pi_{1}\left(L_{0}\right) \rightarrow \pi_{1}(M)$ of the inclusion $i: L_{0} \rightarrow M$ is injective and that $\operatorname{gr}\left(\pi_{1}\left(L_{0}\right)\right) \geq \operatorname{gr}\left(\pi_{1}(L)\right)$ for any leaf $L \in \mathcal{F}$, then $\mathcal{F}$ is without holonomy.

Proof. Take a compact subset $K:=\bigvee_{k} S^{1} \subset L_{0}$ with a base point $x$ for the root of this bouque such that the homomorphism $\pi_{1}(K, x) \rightarrow \pi_{1}\left(L_{0}, x\right)$ induced by the inclusion $K \rightarrow L_{0}$ is surjective. Let $F: K \times[0,1] \rightarrow M$ be a positive trivial fence on the leaf $L_{0}, i_{t}: L_{t} \rightarrow M$ inclusions, $F_{t}:(K, x) \rightarrow\left(L_{t}, x_{t}\right)$ the induced maps by $i_{t} \circ F_{t}=F(\cdot, t)$, and $H_{t}:=\operatorname{im}\left(F_{t *}\right) \leq \pi_{1}\left(L_{t}, x_{t}\right)$ subgroups for any $t \in[0,1]$. By the definitions and the injectivity, $\operatorname{gr}\left(\pi_{1}\left(L_{0}, x_{0}\right)\right)=\operatorname{gr}\left(H_{0}\right)=$ $\operatorname{gr}\left(\operatorname{im}\left(F_{0 *}\right)\right)=\operatorname{gr}\left(i_{0 *}\left(\operatorname{im}\left(F_{0 *}\right)\right)\right)=\operatorname{gr}\left(\operatorname{im}\left(\left(i_{0} \circ F_{0}\right)_{*}\right)\right)$. Since $\operatorname{im}\left(i_{0} \circ F_{0}\right)=F(K, 0)$ and $\operatorname{im}\left(i_{t} \circ F_{t}\right)=F(K, t)$ are free homotopic in $M$, we have $\operatorname{gr}\left(\operatorname{im}\left(\left(i_{0} \circ F_{0}\right)_{*}\right)\right)=$ $\operatorname{gr}\left(\operatorname{im}\left(\left(i_{t} \circ F_{t}\right)_{*}\right)\right)=\operatorname{gr}\left(i_{t *}\left(\operatorname{im}\left(F_{t *}\right)\right)\right)=\operatorname{gr}\left(i_{t *}\left(H_{t}\right)\right) \leq \operatorname{gr}\left(H_{t}\right) \leq \operatorname{gr}\left(\pi_{1}\left(L_{t}, x_{t}\right)\right)$ for any $t \in[0,1]$. By the hypothese, we obtain $\operatorname{gr}\left(\pi_{1}\left(L_{t}, x_{t}\right)\right) \leq \operatorname{gr}\left(\pi_{1}\left(L_{0}, x_{0}\right)\right)$ for any $t \in[0,1]$. Thus $H_{t}$ and $\pi_{1}\left(L_{t}\right)$ have the same polynomial growth. By Lemma 2.5 we have that for any $t \in[0,1]$ and any $g \in \pi_{1}\left(L_{t}\right)$, there exists some $n \in \mathbb{Z}_{>0}$ such that $g^{n} \in \mathrm{im} F_{t *}$. Since any codimension one transversely orientable foliation has no finite holonomy, the leaves in the saturation of $F(K \times] 0,1[)$ are without holonomy. By the minimality of $\mathcal{F}$, we obtain the saturation is $M$. Thus $\mathcal{F}$ is without holonomy.

Now we prove the main theorem.

Proof of Theoren 1.1. Since the union of the leaves without holonomy is dense $\mathrm{G}_{\delta}$ EMT77, there is a leaf $L$ of $\mathcal{F}$ without holonomy. Since $\mathcal{F}$ has no vanishing cycles, by Theorem 3.4. p.147 [HH83], we obtain that the homomorphism $\pi_{1}(L) \rightarrow \pi_{1}(M)$ induced by the inclusion $L \rightarrow M$ is injective. By Lemma 3.1, we have $\mathcal{F}$ is without holonomy.

\section{REMARKS}

The condition that all fundamental groups of leaves have the same polynomial growth is necessary because Anosov foliations for flows satisfy the conditions except this growth condition and have nontrivial holonomy.

The following example shows that the minimal condition in the main theorem is necessary. Consider a continuous map $f:[0,1] \rightarrow[0,1]$ such that $f(0)=0, f(1)=$ 1 , and $f(x)>x$ for any $x \in] 0,1$ [. Identifying 0 with 1 , we obtain the map induced on $S^{1}$ by $f$. Take a suspension foliation $\mathcal{F}_{1}$ on $T^{2}$ of the induced map. Then $\mathcal{F}_{2}=\left\{L \times \mathbb{S}^{1} \mid L \in \mathcal{F}_{1}\right\}$ is a foliation on $T^{3}$ which consists of one torus and other cylinders. Remove the simple closed curve in the toral leaf parallel to the suspended direction. Then the resulting cylindrical leaf has nontrivial holonomy and the resulting non-minimal foliation consists of only cylinders. 
However we do not know whether the minimal condition is necessary for compact manifolds.

\section{REFERENCES}

[EMT77] D. B. A.Epstein, K. C. Millett, D. Tischler, Leaves without holonomy. J. London Math. Soc. (2) 16 (1977), no. 3, 548-552.

[G81] M. Gromov, Groups of polynomial growth and expanding maps Inst. Hautes Etudes Sci. Publ. Math. 53 (1981), 5373.

[HH83] G.Hector, U. Hirsch, Introduction to the geometry of foliations. Part B. Foliations of codimension one. Aspects of Mathematics, E3. Friedr. Vieweg \& Sohn, Braunschweig, 1983.

[N65] S. P. Novikov, Topology of foliations Trudy Moskov. Mat. Obshch. 14 (1965) 248-278.

[Y09] T. Yokoyama, Codimension one minimal foliations and the higher homotopy groups of leaves C. R. Acad. Sci. Paris, Ser. I 347 (2009) 655-658.

[YT08] T. Yokoyama, T. Tsuboi, Codimension one foliations and the fundamental groups of leaves Annales de L'Institut Fourier. (2008), vol. 58, no.2, 723-731.

E-mail address: yokoyama@math.sci.hokudai.ac.jp

Creative Research Institution, Hokkaido University, North 21, West 10, Kita-ku, SAPPORO 001-0021, JAPAN 